\title{
The Formation of Recent and Remote Memory Is Associated with Time-Dependent Formation of Dendritic Spines in the Hippocampus and Anterior Cingulate Cortex
}

\author{
Leonardo Restivo, ${ }^{1,2 \star}$ Gisella Vetere, ${ }^{1,2 *}$ Bruno Bontempi, ${ }^{3}$ and Martine Ammassari-Teule ${ }^{1,2}$ \\ ${ }^{1}$ Istituto di Neuroscienze del Consiglio Nazionale delle Ricerche, and ${ }^{2}$ Istituto di Ricovero e Cura a Carattere Scientifico, Fondazione Santa Lucia, 00143 \\ Rome, Italy, and ${ }^{3}$ Centre de Neurosciences Intégratives et Cognitives, CNRS UMR 5228, Université de Bordeaux 1 et 2, 33405 Talence, France
}

\begin{abstract}
Although hippocampal-cortical interactions are crucial for the formation of enduring declarative memories, synaptic events that govern long-term memory storage remain mostly unclear. We present evidence that neuronal structural changes, i.e., dendritic spine growth, develop sequentially in the hippocampus and anterior cingulate cortex (aCC) during the formation of recent and remote contextual fear memory. We found that mice placed in a conditioning chamber for one 7 min conditioning session and exposed to five footshocks (duration, $2 \mathrm{~s}$; intensity, $0.7 \mathrm{~mA}$; interstimulus interval, $60 \mathrm{~s}$ ) delivered through the grid floor exhibited robust fear response when returned to the experimental context $24 \mathrm{~h}$ or $36 \mathrm{~d}$ after the conditioning. We then observed that their fear response at the recent, but not the remote, time point was associated with an increase in spine density on hippocampal neurons, whereas an inverse temporal pattern of spine density changes occurred on aCC neurons. At each time point, hippocampal or aCC structural alterations were achieved even in the absence of recent or remote memory tests, thus suggesting that they were not driven by retrieval processes. Furthermore, ibotenic lesions of the hippocampus impaired remote memory and prevented dendritic spine growth on aCC neurons when they were performed immediately after the conditioning, whereas they were ineffective when performed $24 \mathrm{~d}$ later. These findings reveal that gradual structural changes modifying connectivity in hippocampal-cortical networks underlie the formation and expression of remote memory, and that the hippocampus plays a crucial but time-limited role in driving structural plasticity in the cortex.
\end{abstract}

\section{Introduction}

One influential theory of memory consolidation posits that the storage and the retrieval of recent memory depends on the hippocampus and that, at later stages, the hippocampus interacts with neocortical sites where corticocortical connections progressively develop and, ultimately, self-govern the storage and the retrieval of remote memory (Squire and Alvarez, 1995; Squire et al., 2004; Frankland and Bontempi, 2005; Squire and Bayley, 2007). To date, experimental evidence supporting this model comes from studies revealing sequential activation of hippocampal and medial prefrontal cortex (mPFC) regions by means of glucose uptake imaging (Bontempi et al., 1999), immediate early genes induction (Frankland et al., 2004), NMDA activity (Takehara-Nishiuchi et al., 2006), or expression of proteins involved in axonal growth and sprouting (Routtenberg et al., 2000;

Received Feb. 26, 2009; revised May 19, 2009; accepted May 20, 2009.

This work was supported by Consiglio Nazionale delle Ricerche (M.A.-T.), Filas Regione Lazio (M.A.-T.), Fondazione Santa Lucia (L.R., G.V.), Fondation Simone et Cino del Duca (B.B.), I'Agence Nationale pour la Recherche (B.B.), and CNRS UMR 5228 (B.B.). We thank Paul W. Frankland, James L. McGaugh, Thomas P. Durkin, Edith Lesburguères, and Hélène Marie for comments on earlier drafts of this manuscript.

*L.R. and G.V. contributed equally to this work.

Correspondence should be addressed to Martine Ammassari-Teule, Istituto di Ricovero e Cura a Carattere Scientifico, Fondazione Santa Lucia, Istituto di Neuroscienze del Consiglio Nazionale delle Ricerche, Via del Fosso di Fiorano 64, 00143 Rome, Italy. E-mail: martine.teule@cnr.it.

L. Restivo's present address: Program in Neurosciences and Mental Health, The Hospital for Sick Children, Toronto, 0N M5G 1X8, Canada.

DOl:10.1523/JNEUROSCI.0966-09.2009

Copyright $\odot 2009$ Society for Neuroscience $\quad$ 0270-6474/09/298206-09\$15.00/0
Frankland et al., 2004; Maviel et al., 2004; Frankland and Bontempi, 2005). These findings support, therefore, the view that consolidation of remote memory requires extra-hippocampal structures (Teng and Squire, 1999; Rosenbaum et al., 2000) and that transformation of initially vulnerable traces into resistant ones involves the coordinated activation of hippocampal and distributed cortical areas including prefrontal, anterior cingulate, and retrosplenial cortices.

Memories, however, are not to be seen as being literally transferred from the hippocampus to the neocortex as they consolidate over time. Several models have proposed that the hippocampus plays a privileged role in organizing remote memory storage that would consist in actively modifying connectivity in distributed cortical networks (McClelland et al., 1995; Squire and Alvarez, 1995). This role intuitively suggests that changes in the morphology of hippocampal or cortical neurons should take place during the formation of recent or remote memories. Insofar, there is evidence that synaptic rearrangements rapidly achieved through an increase in spine density on hippocampal cell dendrites occur at early stages of memory formation (Leuner et al., 2003; Knafo et al., 2004; Restivo et al., 2006). However, whether such rearrangements occur in cortical regions during remote memory formation is unknown.

To address this issue, we trained mice for contextual fear conditioning and processed their brain for Golgi-Cox impregnation at a recent $(24 \mathrm{~h})$ or a remote $(36 \mathrm{~d})$ memory time point. Here, we show that structural plasticity, i.e., dendritic spine growth, se- 
quentially develops in the hippocampus and the anterior cingulate cortex $(\mathrm{aCC})$ during the formation of recent and remote contextual fear memories. The increase in spines was achieved even in the absence of the memory tests, suggesting that off-line neural activity triggered by initial learning was sufficient to promote time- and region-specific structural changes. Ultimately, hippocampal lesions performed shortly, but not late, after the conditioning prevented spine density changes in aCC neurons and impaired remote memory, thus pointing to a crucial but time-limited role for the hippocampus in driving structural plasticity in the cortex.

\section{Materials and Methods}

Animals. A total of 118 male C57BL/6J@Ico mice purchased from Charles River Italy (Calco) were used. At the beginning of the experiments, mice were 9 weeks old, and their weight ranged from 24 to $26 \mathrm{~g}$. They were housed five per cage and maintained in a temperaturecontrolled facility $\left(22 \pm 1^{\circ} \mathrm{C}\right)$ on a $12 \mathrm{~h}$ light/dark cycle with ad libitum access to food and water. All experimental procedures were conducted in accordance with the official European guidelines for the care and use of laboratory animals (86/609/EEC).

Contextual fear-conditioning protocol. Mice were first handled for $3 \mathrm{~d}$ in the vivarium and then for 2 additional days in the experimental room. Contextual fear conditioning consisting of one single session of $7 \mathrm{~min}$ began on the next day. Each mouse was placed in a transparent Plexiglas cage $(28 \times 28 \times 10 \mathrm{~cm})$ with a removable grid floor made of stainless steel rods. After $120 \mathrm{~s}$ of free exploration, the mouse was exposed to a series of five non-signaled footshocks (duration, $2 \mathrm{~s}$; intensity, $0.7 \mathrm{~mA}$; interstimulus interval, $60 \mathrm{~s}$ ) delivered through the grid floor. Control mice were treated identically, except that they were not shocked. Contextual fear memory tests were run by placing the mice back in the conditioning chamber for $4 \mathrm{~min}$, either $24 \mathrm{~h}$ (recent memory) or $36 \mathrm{~d}$ (remote memory) after the conditioning session. Behavior during conditioning or testing was recorded using an automated procedure described previously (Anagnostaras et al., 2000). Briefly, activity was recorded by means of a video camera mounted $60 \mathrm{~cm}$ above the ceiling of the cage and connected to a computer equipped with the Ethovision software (Noldus). Activity suppression ratios and percentage of time spent freezing (absence of all but respiratory movements) were used to score fear memory. The following formula was used to calculate activity suppression ratios: activity testing/(activity conditioning plus activity testing) (the lower the ratio, the better the memory).

Golgi-Cox staining and tissue preparation. Golgi-Cox staining was used to assess postconditioning changes in neuronal morphology at the recent and remote memory time points. Mice were deeply anesthetized with chloral hydrate $(400 \mathrm{mg} / \mathrm{kg}$, i.p.) and transcardially perfused with a solution of $0.9 \%$ saline. Brains were dissected and impregnated using a Golgi-Cox solution, according to the method described by Glaser and Van der Loos (1981). Briefly, they were first immersed in the Golgi-Cox solution at room temperature for $6 \mathrm{~d}$, transferred to a $30 \%$ sucrose solution for $2 \mathrm{~d}$, and then sectioned using a vibratome. Coronal sections (100 $\mu \mathrm{m}$ thick) were mounted on gelatinized slides, stained according to the method described by Gibb and Kolb (1998), and coverslipped with Permount.

Quantification of neuronal morphology and imaging. Spine density was measured on pyramidal neurons located in the CA1 region of the dorsal hippocampus and in layers II/III of the anterior cingulate and of primary visual cortices that has been shown not to be involved in contextual fear memory. These structures were defined according to the Franklin and Paxinos (2001) mouse atlas (see supplemental Fig. 1, available at www. jneurosci.org as supplemental material). Neurons, identified with a light microscope (Leica; DMLB) under low magnification $[\times 20$; numerical aperture (NA), 0.5], were chosen by first locating, among all the stained sections, the regions of interest in their respective coronal sections. Three neurons showing at least fourth-order branches for both apical and basal dendrites in each region and within each hemisphere were selected. Since no significant interhemispheric difference was observed, measurements were pooled so that six neurons per region were studied in each animal.
Only neurons which satisfied the following criteria were chosen for analysis in each of the experimental groups: (1) presence of untruncated dendrites, (2) consistent and dark impregnation along the entire extent of all of the dendrites, and (3) relative isolation from neighboring impregnated neurons to avoid interference and ensure accuracy of dendritic spine counting. Subsequently, dendritic spines were analyzed under a higher magnification $(\times 63$; NA, 0.75$)$. Series of sequential photomicrographs of dendritic segments were generated on a computer screen by means of a video camera connected to the microscope. These photomicrographs were acquired using at least five serial focal planes $(2-3 \mu \mathrm{m}$ apart) by focusing in and out with the fine adjustment of the microscope to create a stack of sequential images. This ensured the accurate reconstruction of entire dendritic segments and enabled counting of these segments with all their visible spines on two-dimensional images. To further minimize any missing of spines, both apical and basal dendrites of the selected segments had to appear as much as possible in the series of focal planes selected for counting. Spines were counted on secondary and tertiary branches of apical dendrites in the stratum radiatum and on secondary and tertiary branches of basal dendrites in the stratum oriens of the CA1 hippocampal field. The same categories of dendrites were analyzed on aCC and visual cortex (VC) pyramidal neurons lying in the II/III layer (see supplemental Fig. 1, available at www.jneurosci.org as supplemental material). On each neuron and for each dendrite category, five $20 \mu \mathrm{m}$ dendritic segments were randomly selected using a twodimensional sampling grid made of $20 \mu \mathrm{m}$ squares to generate values of spine density (see supplemental Fig. 1, available at www.jneurosci.org as supplemental material). In some cases, segments were from the same branch (Leuner et al., 2003). Segments were sampled $50 \mu \mathrm{m}$ away from soma to exclude the spine-depleted zone which arises from the cell body. Only protuberances with a clear connection of the head of the spine to the shaft of the dendrite were counted as spines. Since this method has proven to provide reliable results (Horner and Arbuthnott, 1991), no attempt was made to introduce a correction factor for hidden spines. As no difference in spine counts was observed between secondary and tertiary branch segments for each group, data were pooled for each dendrite category (basal and apical) to generate the final spine density results. All measurements were performed by an experimenter blind to the experimental conditions.

Spine density measurements after recent and remote memory tests. Mice $(N=40)$ were subjected to contextual fear conditioning $(N=20)$ or pseudoconditioning $(N=20)$. In each subgroup, half of the mice were tested for recent or remote memory, respectively, $24 \mathrm{~h}$ or $36 \mathrm{~d}$ after the conditioning episode. Twenty-four hours after the completion of the retention tests, their brains were processed for Golgi staining. Dendritic spines were counted on pyramidal neurons in the CA1 hippocampal subfield, the anterior cingulate cortex, and the primary visual cortex.

Spine density measurements in mice that were not subjected to memory tests. Mice $(N=20)$ were conditioned $(N=10)$ or pseudoconditioned $(N=10)$ without undergoing recent or remote memory tests. In each subgroup, half of the mice were killed $48 \mathrm{~h}$ after the conditioning and the other half $37 \mathrm{~d}$ later and their brains processed for Golgi staining. Dendritic spines were counted on CA1 (recent time point) and anterior cingulate cortex (remote time point) pyramidal neurons.

Effect of early or late postconditioning hippocampal lesions on remote memory and cortical spine density. Mice $(N=58)$ were conditioned or pseudoconditioned and then randomly allocated to early $(N=32)$ or late $(N=26)$ lesion groups. Mice in these four groups were further subdivided in mice receiving lesions (early, $N=20$; late, $N=16$ ) or sham lesions (early, $N=12$; late, $N=10$ ). Early lesions were performed immediately after the conditioning and late lesions $24 \mathrm{~d}$ later. Hippocampal lesions were produced by inserting stainless steel cannulae bilaterally in the dorsal hippocampus using the following stereotaxic coordinates: anteroposterior relative to bregma, $-2.1 \mathrm{~mm}$; lateral to midline, $\pm 2.1 \mathrm{~mm}$; ventral from the skull surface, $-1.8 \mathrm{~mm}$. The excitotoxic agent ibotenic acid $(0.2 \mu \mathrm{l}$ per side over $2 \mathrm{~min})$ was slowly infused bilaterally using a perfusion pump connected to the cannulae. Sham-lesioned mice underwent the same procedure, except that sterile saline $(0.9 \% \mathrm{NaCl})$ was infused. After completion of early or late surgery, mice were returned to their home cage and tested for remote memory $36 \mathrm{~d}$ after the condition- 


\section{Activity suppression}

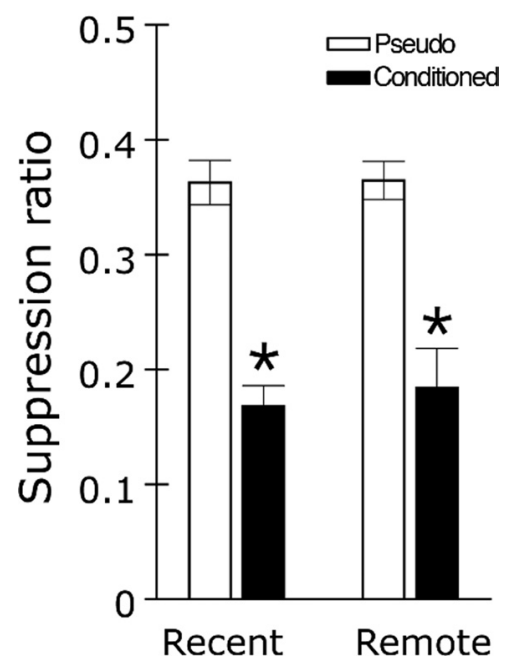

Figure 1. Contextual fear conditioning elicits a robust fear response $24 \mathrm{~h}$ or $36 \mathrm{~d}$ after the conditioning. Conditioned mice (black bars) showed lower activity suppression ratios during the recent and remote memory tests compared with pseudoconditioned mice (white bars) that were not shocked. No forgetting occurred across retention intervals. ${ }^{*} p<0.05 ; N=10$ mice per group.

ing. Twenty-four hours after the remote memory test, their brains were processed for morphological analyses. After the perfusion, the anterior part of the brain was Golgi stained to count dendritic spines on aCC neurons, whereas the posterior part of the brain was stained according to the Nissl method to estimate hippocampal neuronal loss attributable to ibotenic acid lesions. Only mice with cannula tips correctly located, and whose extent of lesion was circumscribed to the dorsal hippocampus, were included in this study. Accordingly, six early lesioned mice out of 20 (three conditioned and three pseudoconditioned) and five late-lesioned mice out of 16 (two conditioned and three pseudoconditioned) were excluded.

Statistical analyses. Results were expressed as means \pm SEM. Retention performance (activity suppression and freezing) was compared across groups by means of a two-way ANOVA with training condition (conditioned, pseudoconditioned) and retention interval (recent, remote) as main factors. Differences in spine density were assessed by means of a three-way ANOVA with training condition (conditioned, pseudoconditioned, naive), retention interval (recent, remote), and brain region (CA1, aCC, VC) as main factors. Post hoc analyses were performed using the Fisher's protected least significant differences test. Cumulative frequencies of spine density in conditioned, pseudoconditioned, and naive mice were compared across groups using, first, the normal distribution Kolmogorov-Smirnov (K-S) fitting test and then K-S two-sample tests for subsequent paired comparisons. Differences in spine density between conditioned and pseudoconditioned mice that were not subjected to the retention tests were estimated in the hippocampus at the recent time point and in the aCC at the remote time point by means of Student's $t$ tests. The effect of hippocampal lesions on remote memory and spine density in the aCC was evaluated by means of a two-way ANOVA with training condition (conditioned, pseudoconditioned) and treatment (lesioned, sham lesioned) as main factors. Separate analyses were performed for mice receiving early or for late lesions. Values of $p<0.05$ were considered as significant.

\section{Results}

Conditioned mice show robust contextual fear responses during the recent and the remote memory test

Mice exposed to the footshocks showed greater activity suppression ratios (training condition effect, $F_{(1,36)}=63.80 ; p<0.0001$ ) (Fig. 1) and freezing responses (training condition effect, $F_{(1,36)}$
$=1595.86 ; p<0.0001$ ) (supplemental Fig. 2, available at www. jneurosci.org as supplemental material) relative to non-shocked mice at each retention interval. No forgetting occurred across retention intervals (retention interval effect, $p>0.5$ for each variable).

\section{Contextual fear conditioning promotes a time-dependent increase in dendritic spine density in the CA1 hippocampal region and the anterior cingulate cortex}

Spine density was measured on apical and basal dendrites of CA1, $\mathrm{aCC}$, and VC neurons at each retention interval in conditioned, pseudoconditioned, and naive mice. Results are shown in Figure 2. Structural rearrangements that were specific to the conditioned mice developed sequentially in the hippocampus and the aCC during the formation of recent and remote memory (training condition $\times$ retention interval $\times$ brain region interaction, $F_{(2,48)}=4.36 ; p<0.05$ for apical dendrites and $F_{(2,48)}=5.22 ; p<$ 0.01 for basal dendrites). Post hoc comparisons of spine density in the hippocampus revealed that conditioned mice exhibited a significant increase in spines on apical (spines/20 $\mu \mathrm{m}, 16.6 \pm 0.21$; $p<0.01$ ) and basal (spines/20 $\mu \mathrm{m}, 16.9 \pm 0.42$; $p<0.01$ ) dendrites of CA1 neurons compared with pseudoconditioned mice (apical, spines/20 $\mu \mathrm{m}: 14.9 \pm 0.81$; basal, spines/20 $\mu \mathrm{m}: 14.7 \pm$ 0.84 ) after the recent memory test (Fig. 2a). This increase, however, was only transient, since hippocampal spine values counted after the remote memory test were similar in conditioned (apical, spines $/ 20 \mu \mathrm{m}$ : $11.7 \pm 0.61$; basal, spines $/ 20 \mu \mathrm{m}$ : $11.6 \pm 0.36$; raw data) and pseudoconditioned mice (apical, spines $/ 20 \mu \mathrm{m}: 10.9 \pm$ 0.27; basal, spines $/ 20 \mu \mathrm{m}$ : $11.4 \pm 0.32$ ) (Fig. $2 b$ ). Interestingly, a significant increase $(p<0.05)$ in spine density was found in the hippocampus of pseudoconditioned mice (apical, spines $/ 20 \mu \mathrm{m}$ : $14.9 \pm 0.81$; basal, spines $/ 20 \mu \mathrm{m}: 14.7 \pm 0.84)$ compared with naive mice (apical, spines $/ 20 \mu \mathrm{m}: 12.8 \pm 0.57$; basal, spines $/ 20$ $\mu \mathrm{m}: 11.5 \pm 0.84)$ at the short retention interval, possibly reflecting the encoding of a nonaversive contextual representation. Post hoc comparisons of spine density in the aCC indicated an inverted temporal pattern of structural changes. At the recent time point, spine density values were in the same range in conditioned (apical, spines/20 $\mu \mathrm{m}$ : $14.9 \pm 0.23$; basal, spines/20 $\mu \mathrm{m}$ : $13.4 \pm$ 0.13 ) and pseudoconditioned mice (apical, spines $/ 20 \mu \mathrm{m}: 15.3 \pm$ 0.09; basal, spines $/ 20 \mu \mathrm{m}: 13.8 \pm 0.13$ ) (Fig. $2 c$ ). These values, however, were significantly higher $(p<0.01)$ in the conditioned (apical, spines/20 $\mu \mathrm{m}$ : $16.7 \pm 0.33$; basal, spines/20 $\mu \mathrm{m}$ : $14.2 \pm$ 0.28 ) than in the pseudoconditioned group (apical, spines $/ 20$ $\mu \mathrm{m}: 14.4 \pm 0.48$; basal, spines $/ 20 \mu \mathrm{m}: 13.1 \pm 0.48$ ) at the remote time point (Fig. $2 d$ ). Post hoc comparisons of spine density in layer II/III of primary VC neurons showed that spine density values were similar in all groups at any interval (Fig. $2 e, f$ ).

\section{Spine density changes in hippocampal and cortical networks are extensive}

Cumulative frequency distribution curves of spines measured on hippocampal, aCC, and VC neuron dendrites and photomicrographs of Golgi-impregnated dendritic segments in each condition are shown in Figure 3. For the hippocampus, a stronger shift of the curves to the right was observed in conditioned mice compared with pseudoconditioned and naive mice at the recent memory time point ( $p<0.01$ for both dendrite categories), a pattern revealing that spines were increased on the majority of sampled neurons. At the same time point, the curves of pseudoconditioned mice also showed a significant shift on the right compared with those of naive mice $(p<0.05$ for both dendrite categories), further indicating that encoding of a nona- 


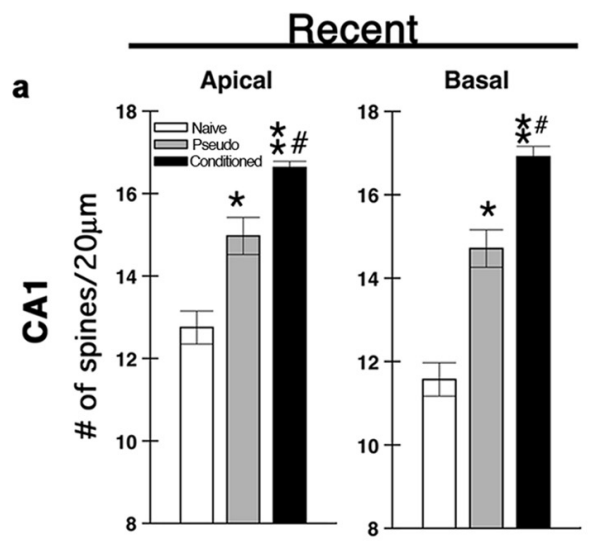

c
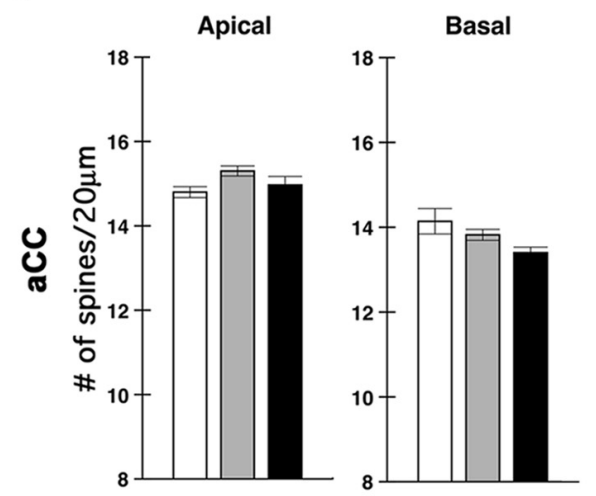

e

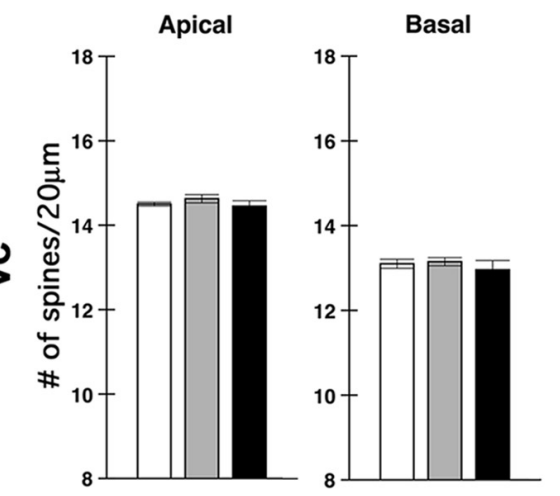

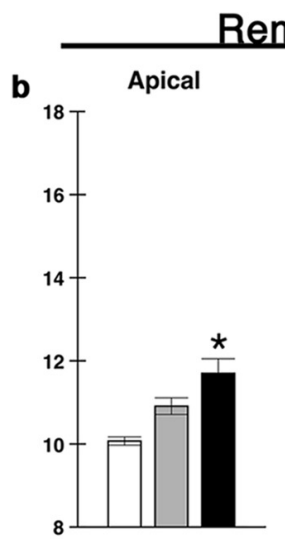

Remote

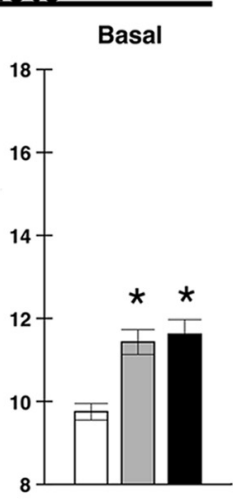

d
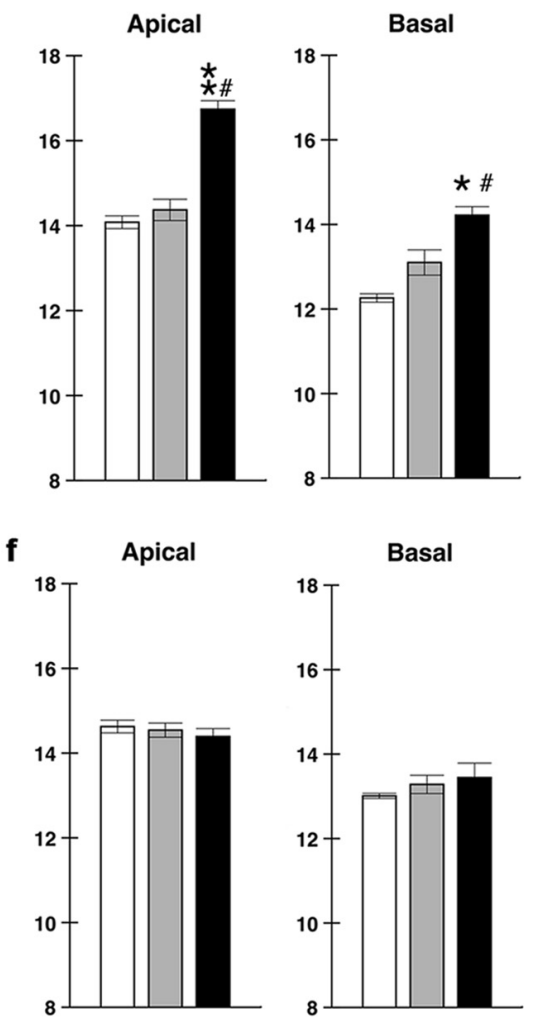

$17.1 \pm 0.60$; basal, spines/20 $\mu \mathrm{m}: 17.3 \pm$ 0.90 ) on hippocampal dendrites $48 \mathrm{~h}$ after they were exposed to the footshocks relative to their pseudoconditioned counterpart (apical, spines $/ 20 \mu \mathrm{m}: 16.4 \pm 0.80$; basal, spines/20 $\mu \mathrm{m}: 14.8 \pm 0.14$ ) (basal, $t_{(8)}=2.4 ; p<0.05$ ) (Fig. 4a,b). Similarly, conditioned mice that were not subjected to the remote memory test showed an increase in spine density on aCC dendrites $37 \mathrm{~d}$ after they were exposed to the footshocks (conditioned: apical, spines/20 $\mu \mathrm{m}: 16.2 \pm 0.15$; basal, spines $/ 20 \mu \mathrm{m}$ : $14.8 \pm 0.24$; pseudoconditioned: apical, spines $/ 20 \mu \mathrm{m}: 14.6 \pm 0.15$; basal, spines $/ 20 \mu \mathrm{m}: 14.0 \pm 0.15$; apical: $t_{(8)}=$ 7.91; $p<0.001)$; basal: $t_{(8)}=2.74 ; p<$ 0.05) (Fig. 4c,d).

\section{The hippocampus drives structural} changes in the cortex, but its role is time limited

The data are shown on Figure 5. Histological controls of ibotenic hippocampal lesions performed immediately (early) or $24 \mathrm{~d}$ (late) after the conditioning revealed similar robust cell loss in CA1, CA3, and dentate gyrus, whereas the subiculum was spared (Fig. 5a,b; supplemental Fig. 3, available at www.jneurosci.org as supplemental material). Statistical analysis performed on activity suppression ratios of mice receiving early lesions revealed a significant effect of the training condition $\left(F_{(1,22)}=35.23 ; p<0.001\right)$ and of the training condition $\times$ treatment interaction $\left(F_{(1,22)}=4.53 ; p<0.05\right)$. Post hoc tests then indicated that conditioned mice with early postconditioning hippocampal lesions showed a higher activity suppression ratio than conditioned mice with sham lesions $(p<0.05)$. No effect of the lesion on this variable was observed in pseudoconditioned mice (Fig. $5 c$ ). In the conditioned mice, the amount of freezing was also significantly lower in the lesioned than in the sham-lesioned group $\left(t_{(11)}=3.57 ; p<\right.$ 0.01) (supplemental Fig. 4, available at www.jneurosci.org as supplemental material). Remarkably, early hippocampal lehippocampal and cortical networks. $\boldsymbol{a}-\boldsymbol{d}$, Retrieval of recent $(\boldsymbol{a})$, but not remote $(\boldsymbol{b})$, memories was associated with an increase in spine density on apical and basal dendrites of pyramidal CA1 neurons, whereas an inverse temporal pattern of structura changes occurred on aCC pyramidal neurons $(\boldsymbol{c}, \boldsymbol{d})$. Spine density of VC pyramidal neurons did not vary between groups at any retention intervals $(\boldsymbol{e}, \boldsymbol{f})$. ${ }^{\#} p<0.01$ versus respective pseudoconditioned controls; ${ }^{*} p<0.05$ and ${ }^{* *} p<0.01$ versus respective naive controls; $N=5$ mice per group.

versive contextual representation elicited structural changes in the hippocampus. For the aCC, a significant shift to the right was observed for the curves of conditioned mice at the remote memory time point ( $p<0.01$ for both dendrite categories). The finding that, at this time point, the curves of pseudoconditioned and naive mice overlapped suggests that cortical remodeling was a net remote memory effect. In the VC, the curves fully overlapped at the recent and remote memory time points.

\section{Structural remodeling of hippocampal-cortical networks is} independent from memory retrieval

Conditioned mice that were not subjected to the recent memory test showed an increase in spine density (apical, spines/20 $\mu \mathrm{m}$ : sions completely abolished late development of dendritic spine growth in aCC neurons in the conditioned mice. Specifically, a significant effect of the training condition $\times$ treatment interaction was found for each dendrite category $\left(F_{(1,19)}=28.74 ; p<\right.$ 0.001 for apical dendrites; $F_{(1,19)}=4.89 ; p<0.05$ for basal dendrites) with post hoc tests showing that conditioned mice receiving sham lesions exhibited more spines on aCC neuron dendrites than the other groups (conditioned plus sham, spines $/ 20 \mu \mathrm{m}$ : $16.1 \pm 0.14$; conditioned plus lesion, spines $/ 20 \mu \mathrm{m}: 14.6 \pm 0.19$; $p<0.01$ for apical dendrites; conditioned plus sham, spines $/ 20$ $\mu \mathrm{m}: 13.5 \pm 0.37$; conditioned plus lesion, spines $/ 20 \mu \mathrm{m}: 12.7 \pm$ $0.21 ; p<0.05$ for basal dendrites) (Fig. $5 d$ ). Also, the shift to the right of cumulative frequency distribution curves found in sham- 
a

\section{Apical}

Recent
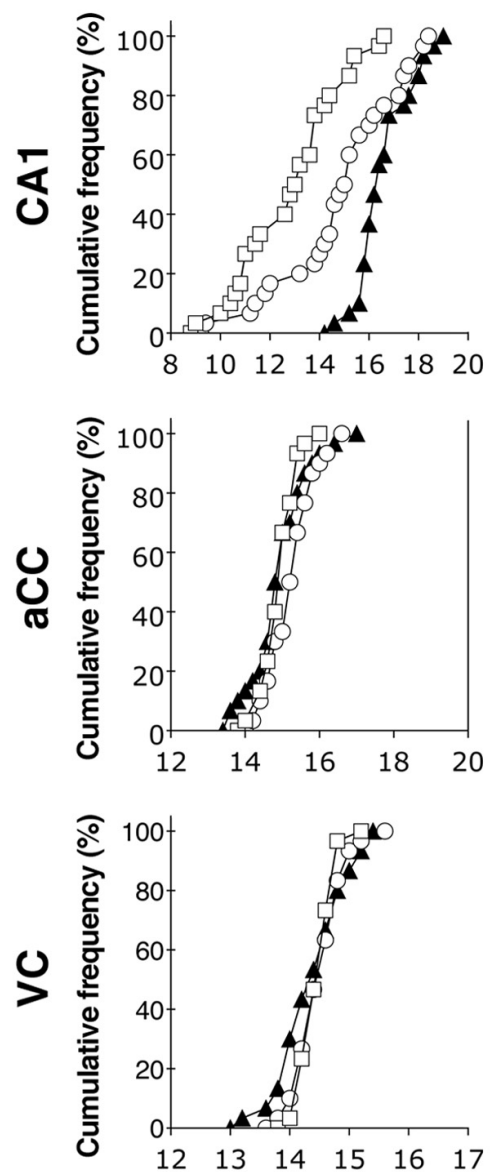

b

\section{Basal}

Recent
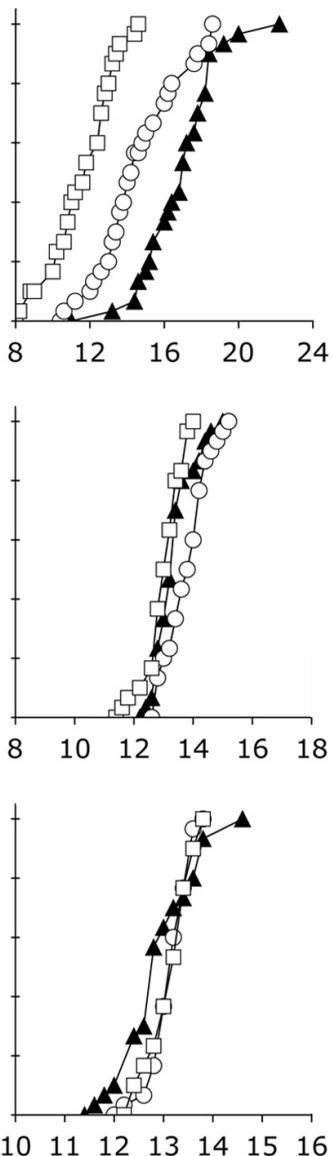

Remote
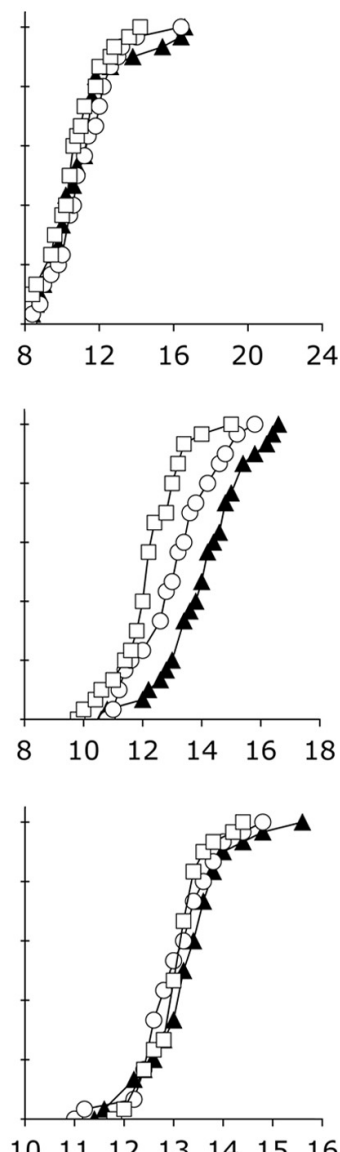

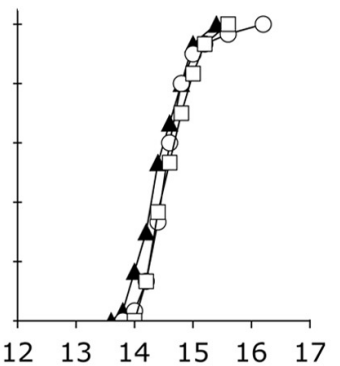

Spine density (spines/20 $\mu \mathrm{m}$ )

Conditioned

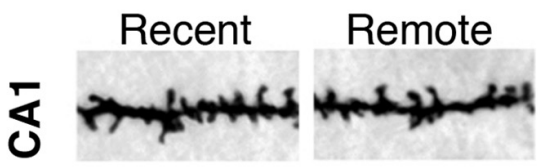

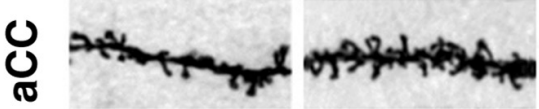

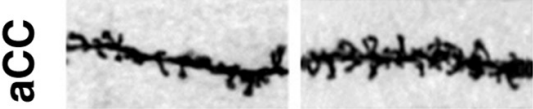

\section{Pseudo}

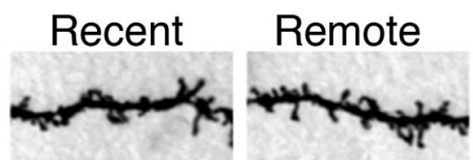

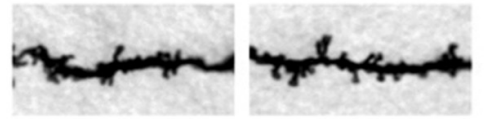

Naive
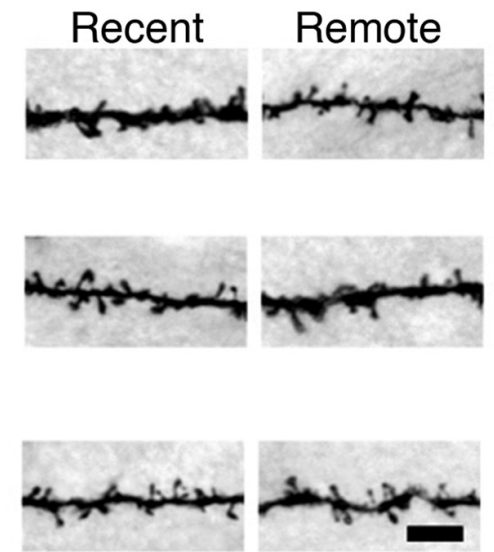

Figure 3. Morphological changes in hippocampal-cortical networks after recent and remote memory tests are extensive. $\boldsymbol{a}$, Cumulative frequency of spine density values along apical and basal dendrites of $\mathrm{CA1}$, aCC, and VC pyramidal neurons are shown for conditioned (filled triangles), pseudoconditioned (open circles), and naive mice (open squares). Each point represents the average spine density (number of spines counted along $20 \mu \mathrm{m}$ dendritic segments) of a single neuron. A shift of the curve to the right indicates that the majority of the sampled neurons showed an increase in spines. Such an increase was found on CA1 neurons at the recent, but not the remote, time point in conditioned mice compared with pseudoconditioned and naive mice. A milder increase was also present in pseudoconditioned mice compared with naive mice. An inverted pattern of structural changes was observed in pyramidal neurons of the aCC where a shift to the right of the cumulative frequency curve of conditioned mice was found at the remote, but not the recent, time point. Cumulative frequency curves in the VC fully overlapped at each time point. $\boldsymbol{b}$, Photomicrographs of Golgi-impregnated basal dendritic segments showing spine density in the CA1 region of the hippocampus, the aCC, and VC after the recent and remote memory tests. Scale bar, $5 \mu \mathrm{m}$. 


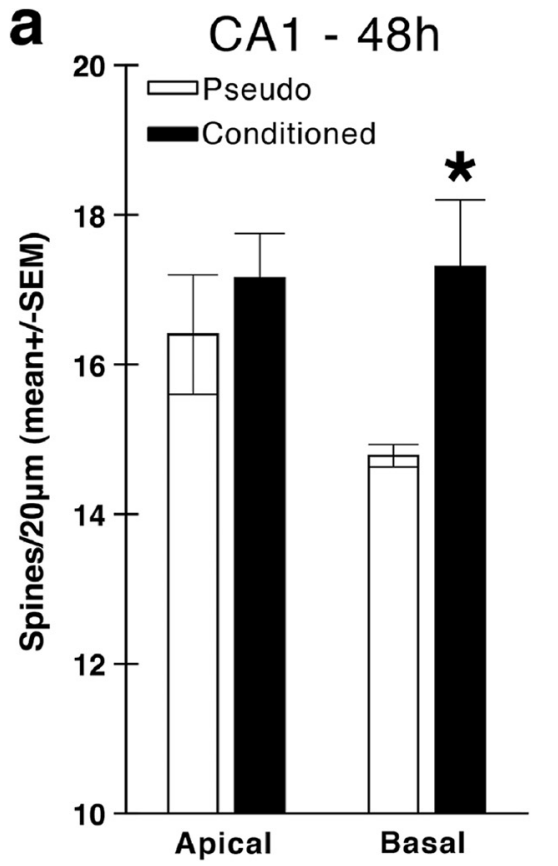

b
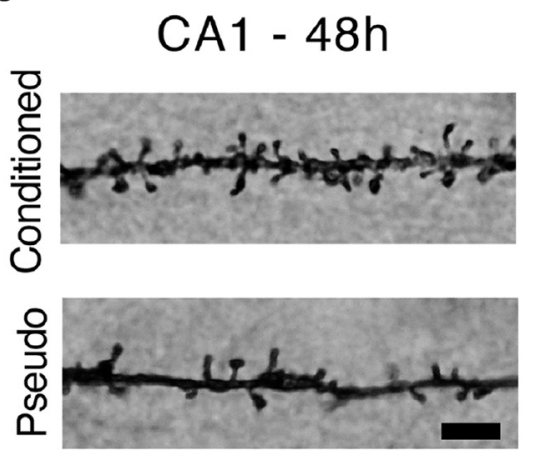

C $\mathrm{aCC}-37 d$

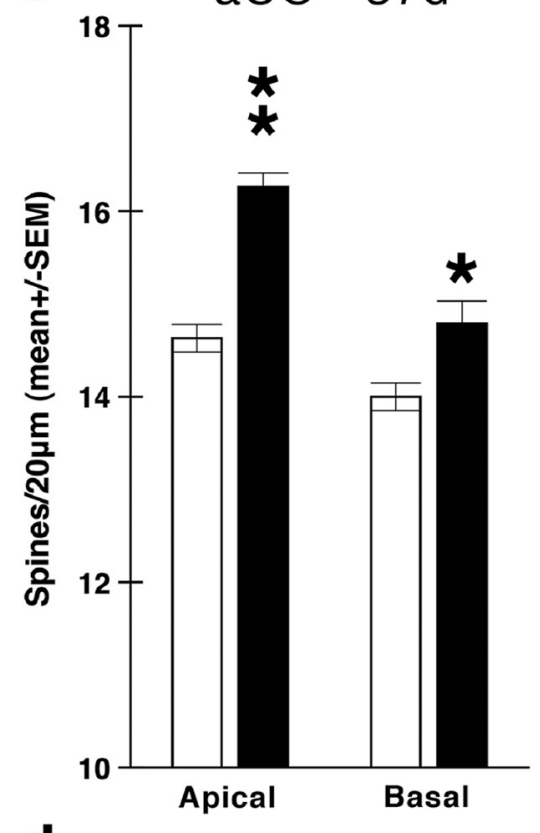

aCc $-37 d$

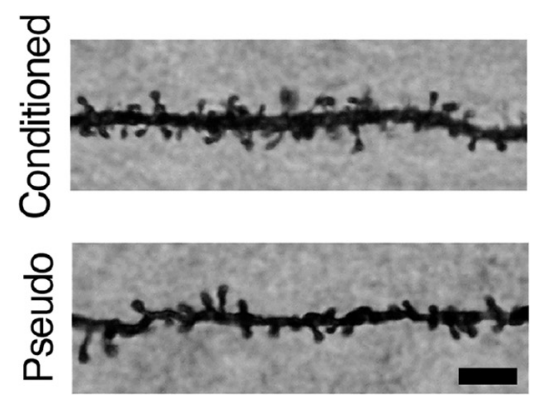

Figure 4. Consolidation-induced synaptic remodeling in hippocampal-cortical networks is independent of retrieval processes. $\boldsymbol{a}$, Conditioned mice that were not subjected to the recent memory test show an increase in spine density on CA1 neuron dendrites $48 \mathrm{~h}$ after the conditioning. $\boldsymbol{b}$, Photomicrographs of Golgi-impregnated apical dendritic segments showing changes in the number of spines counted in the hippocampal CA1 of mouse brains at the same postconditioning interval. Scale bar, $5 \mu \mathrm{m}$. $\boldsymbol{C}$, Conditioned mice that were not subjected to the remote memory test show an increase in spine density on aCC neuron dendrites $37 \mathrm{~d}$ after the conditioning. $\boldsymbol{d}$, Same as $\boldsymbol{b}$ but for the aCC examined $37 \mathrm{~d}$ after the conditioning. Morphological changes observed in CA1 and aCC neurons were similar in magnitude to those observed in mice subjected to retrieval (Figs. 2, 3). ${ }^{*} p<0.05,{ }^{* *} p<$ $0.001 ; N=5$ mice per group.

lesioned conditioned mice relative to pseudoconditioned mice ( $p<0.01$ for apical dendrites; $p<0.05$ for basal dendrites) was completely abolished by the hippocampal lesions (Fig. $6 a, b$ ).

Conversely, mice receiving late postconditioning hippocampal lesions did not show any reduction in activity suppression ratio (Fig. $5 e$ ) or freezing $\left(t_{(9)}=0.91 ; p>0.38\right.$ ) (supplemental Fig. 5, available at www.jneurosci.org as supplemental material) compared with sham-lesioned conditioned mice during the remote memory test, since the analysis revealed only a significant effect of the training condition $\left(F_{(1,17)}=126.00 ; p<0.001\right)$. Late hippocampal lesions did not prevent conditioned mice to show an increase in spine density on aCC neurons (apical dendrites, conditioned plus sham, spines $/ 20 \mu \mathrm{m}: 16.7 \pm 0.44$; conditioned plus lesion, spines/20 $\mu \mathrm{m}$ : $16.2 \pm 0.44$; pseudoconditioned plus sham, spines $/ 20 \mu \mathrm{m}: 14.1 \pm 0.63$; pseudoconditioned plus lesion, spines $/ 20 \mu \mathrm{m}: 13.7 \pm 0.45$; basal dendrites, conditioned plus sham, spines $/ 20 \mu \mathrm{m}: 14.7 \pm 0.45$; conditioned plus lesion, spines $/ 20 \mu \mathrm{m}: 13.5 \pm 0.67$; pseudoconditioned plus sham, spines $/ 20 \mu \mathrm{m}: 12.6 \pm 0.43$; pseudoconditioned plus lesion, spines/20 $\mu \mathrm{m}: 11.6 \pm$ 0.61 ; lesion effect: $F_{(1,17)}>0.83 ; p>0.5$ for both dendrite categories) (Fig. $5 f$ ) or to display a normal pattern of cumulative frequency distribution of spine density (Fig. $6 c, d)$ at the remote time point.

\section{Discussion}

Memory traces are not definitively fixed at the time of encoding but undergo a gradual process of stabilization and consolidation (Dudai, 2004; Squire et al., 2004). Recent memories are thought to be initially dependent on protein synthesis-related modifications of hippocampal synaptic connections (Bailey et al., 2004). Their storage in this brain region is, however, time limited, since damage to the medial temporal lobe, which includes the hippocampus, disrupts recent memory retrieval, whereas remote memories are left unaffected (Zola-Morgan and Squire, 1990; Kim and Fanselow, 1992; Martin and Clark, 2007; Squire and Bayley, 2007). Consistent with this model, we provide direct evidence that time-dependent changes in spine density occur in hippocampal and cortical networks during the formation of recent and remote memory. Specifically, our findings point to a highly dynamic process of memory consolidation triggering complex time- and region-dependent structural rearrangements that involve both the formation and elimination of spines. First, we observed that the formation of a recent aversive memory was associated with an increase in dendritic spines on CA1 pyramidal neurons that was specific to the conditioned mice. Importantly, this increase was found to be transient, since the number of spines counted in the conditioned and pseudoconditioned groups $36 \mathrm{~d}$ after the conditioning was no longer different. Second, a robust fear response to the context was still present at the remote time point and was now associated with an increase in spines on aCC pyramidal cell dendrites. Indeed, whether the aCC actively contributes to memory storage per se or, as hypothesized (Simons and Spiers, 2003; Frankland and Bontempi, 2005), exerts an executive role in integrating and coordinating memories from distributed cortical modules processing selective aspects of the memory trace (Ross and Eichenbaum, 2006) remains to be determined.

A central tenet of the systems memory consolidation theory is that the recruitment of cortical networks in remote memory storage requires early activation of hippocampal-mPFC circuits. According to this view, hippocampal lesions performed shortly after the conditioning would prevent the increase in spines on aCC neurons and disrupt remote memory. We directly tested this prediction by lesioning the hippocampus after completion of the conditioning episode and then examining conditioning performance and cortical morphology $36 \mathrm{~d}$ later. Results showed that 
a

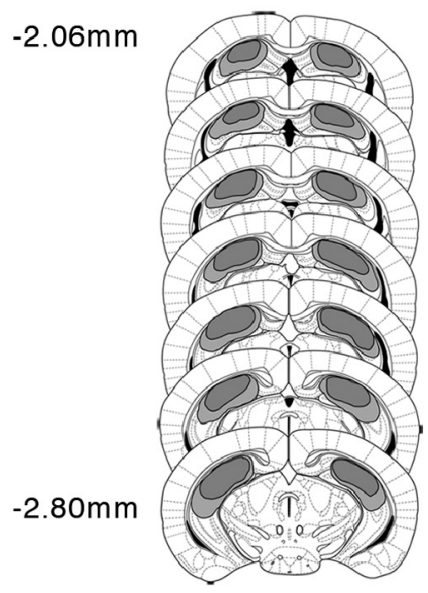

b

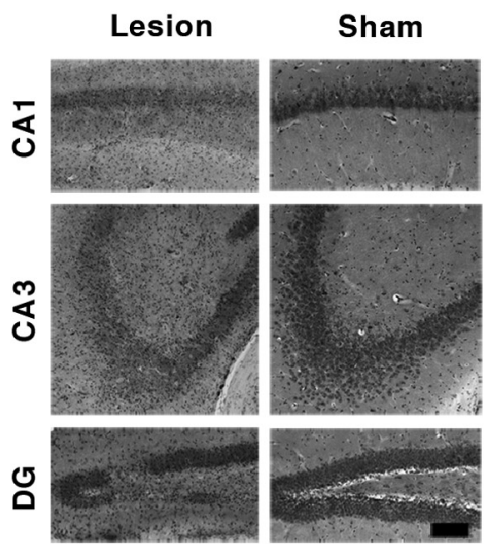

c
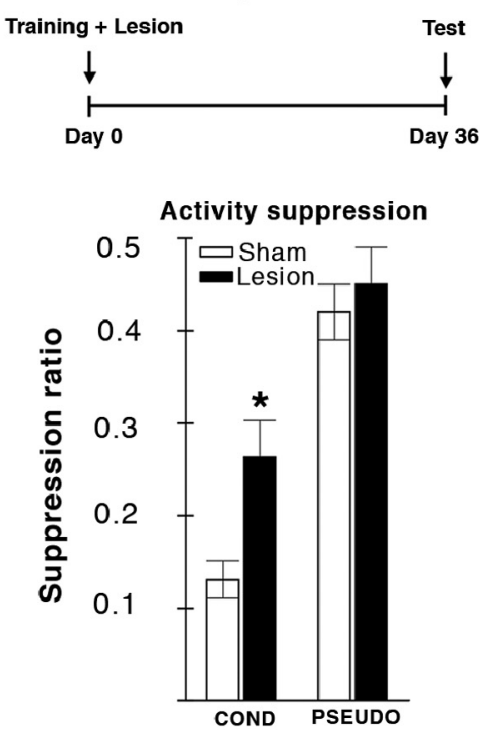

e
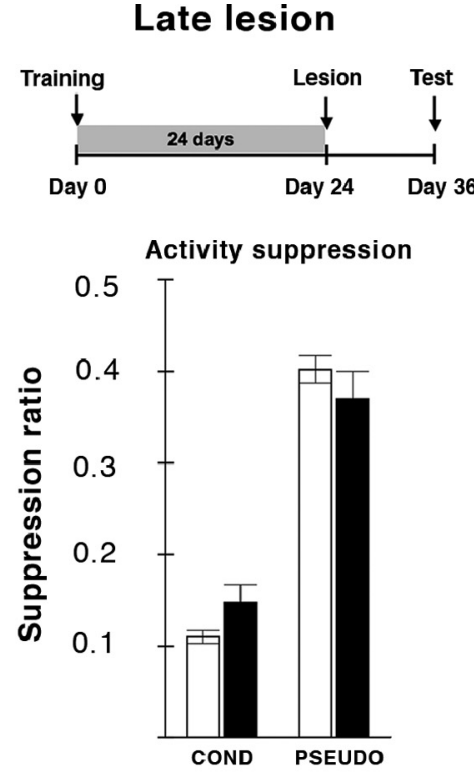

d

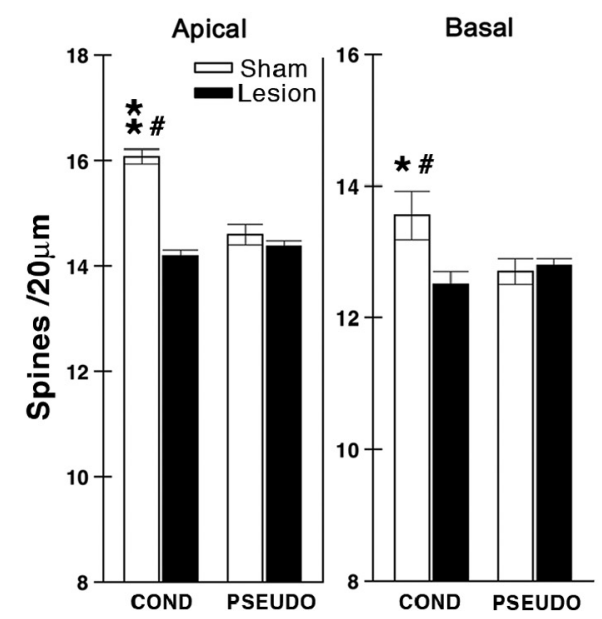

f
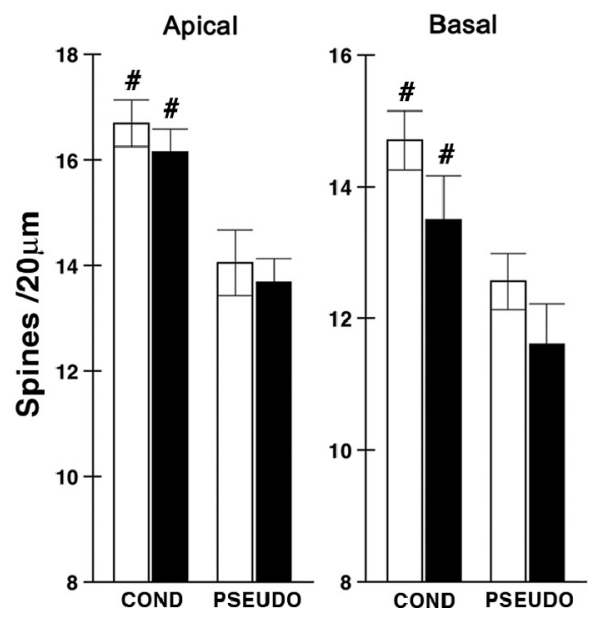

Figure 5. Early, but not late, hippocampal lesions impair remote memory and prevent spine density changes on aCC neurons. $\boldsymbol{a}$, Coronal diagrams of mouse brain sections [adapted from Franklin and Paxinos (2001)] showing the extent of hippocampal lesions (maximal extent is shown in light gray, whereas minimal extent appears darker). Lesions were specific and mainly extended through the $C A 1, C A 3$, and dentate gyrus (DG) fields of the dorsal hippocampus, whereas the subiculum was spared. $\boldsymbol{b}$, Representative photomicrographs of hippocampal tissue in lesioned and sham-lesioned mice. Cresyl violet staining revealed consistent and similar cell shrinkage and microglial activation in the early and late lesion groups (see also supplemental Fig. 3 , available at www.jneurosci.org as supplemental material). Scale bar, $100 \mu \mathrm{m}$. c, Mice with early hippocampal lesions exhibited lower activity suppression ratios than sham-lesioned mice when tested for remote memory. $\boldsymbol{d}$, Early hippocampal lesions (black bar) prevented the increase in spine density on aCC neuron dendrites after the remote memory test, whereas this increase was still present in sham-lesioned mice (white bars). $\boldsymbol{e}$, Mice with late hippocampal lesions showed intact remote memory performance. $\boldsymbol{f}$, Late hippocampal lesions did not prevent the increase in spine density on aCC neuron dendrites after the remote memory test. ${ }^{*} p<0.05$ and ${ }^{* *} p<0.01$ versus lesion group; ${ }^{\#} p<0.05$ versus respective pseudoconditioned sham or lesion controls; $N=5-7$ mice per group.

not only remote memory was impaired, as it could be expected from studies showing retrograde amnesia gradients after hippocampal damage (Squire et al., 2004; Squire and Bayley, 2007) but, more importantly, that hippocampal lesions prevented consolidation-induced spine growth on aCC neurons at the remote time point. Furthermore, to ascertain that remote memory storage requires only a time-limited activation of hippocampalmPFC circuits, we performed hippocampal lesions long after (24 d) the conditioning with the prediction that spine density changes would occur on aCC neurons and remote memory would be spared. The results of our experiment support this prediction, since mice undergoing late lesions showed both robust conditioning and an increase in spines on cortical neurons. Our findings, therefore, show that formation and expression of longlasting memories entail structural plasticity in aCC and that consolidation-induced cortical spine rearrangements require an intact hippocampus only at early stages of memory formation. Indeed, how the hippocampus manages to activate the experience-relevant set of cortical neurons is still a matter of debate, although potential mechanisms, including synaptic tagging, have been proposed (Frey and Morris, 1998; Martin and Kosik, 2002).

To ensure that remodeling of hippocampal and cortical neurons was actually a correlate of contextual fear memory, our mice were previously subjected to recent and remote memory tests raising the possibility that the observed morphological rearrangements were triggered by retrieval processes. To determine whether consolidation per se would yield comparable results, we tested additional groups of mice that did not undergo postconditioning tests. We found that similar time-dependent structural 
a Early lesion
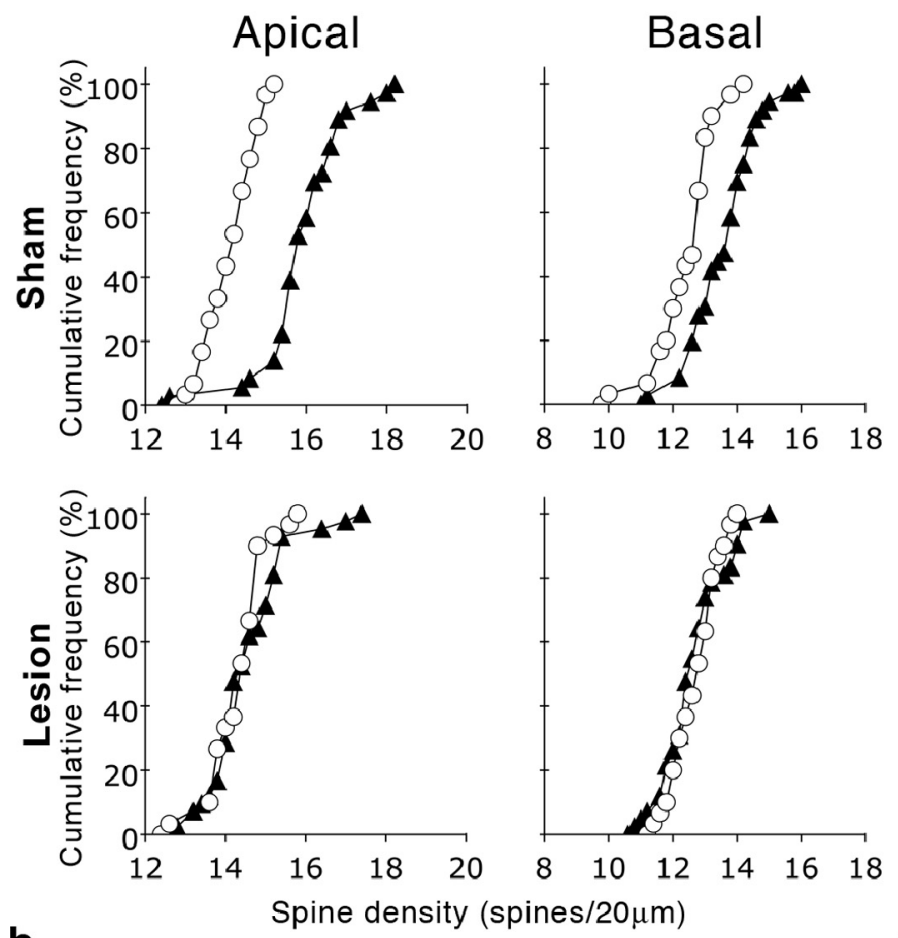

b

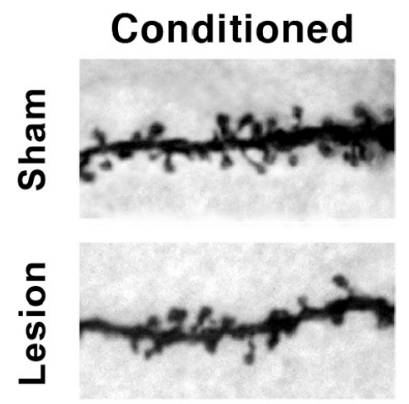

Pseudo
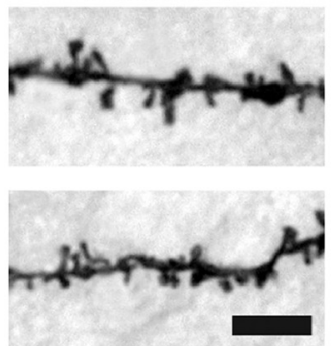

C

Late lesion
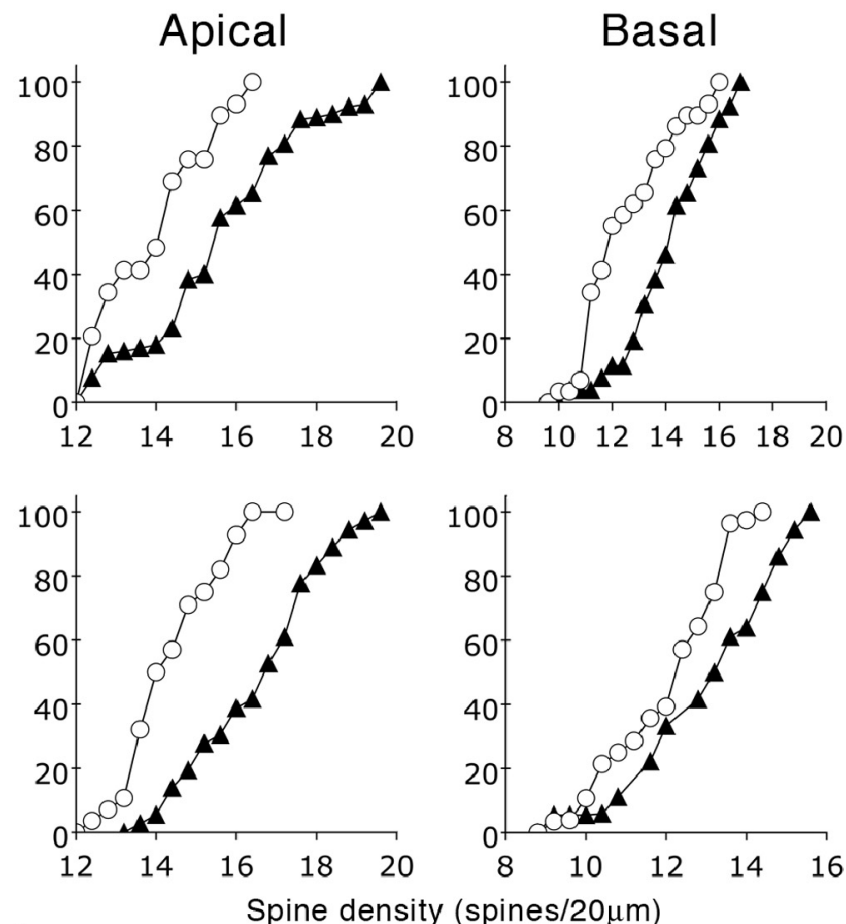

d Spine density (spines $/ 20 \mu \mathrm{m}$ )

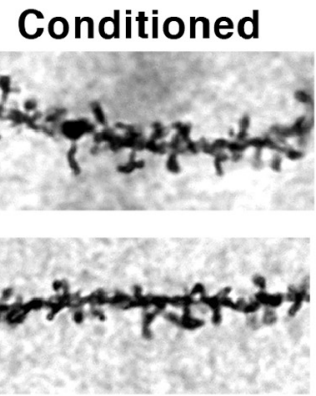

Pseudo

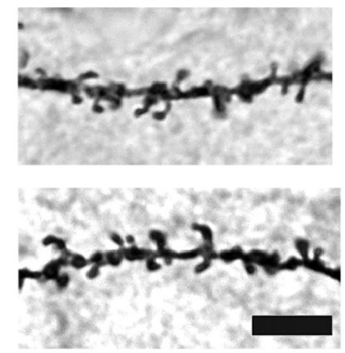

Figure 6. Early hippocampal lesions produce widespread disruption of spine growth in ac( neurons. $\boldsymbol{a}$, A shift to the right of the curves depicting the cumulative frequency of spine density values on apical and basal dendrites of aCC neurons was observed in sham-lesioned conditioned mice (filled triangles) compared with pseudoconditioned mice (open circles), indicative of extensive structural changes in the conditioned group (top). Conversely, spine growth was disrupted on the majority of sampled neurons in early lesioned conditioned mice, as shown the overlapping of their cumulative frequency distribution curves with the one of pseudoconditioned mice $(N=30-42$ neurons per group; bottom). $\boldsymbol{b}$, Photomicrographs of Golgi-impregnated apical dendritic segments of aCC neurons. Scale bar, $5 \mu \mathrm{m}$. c, Late hippocampal lesions did not prevent widespread spine growth in the aCC, since the cumulative frequency distribution curves of conditioned mice were shifted to the right compared with the curves of pseudoconditioned mice; either they were lesioned or sham lesioned ( $N=30-36$ neurons per group). $\boldsymbol{d}$, Same as $\boldsymbol{b}$ but for mice that received late hippocampal lesions.

rearrangements developed in conditioned groups. Thus, off-line neural activity triggered by initial learning alone is sufficient to elicit structural plasticity in the hippocampus and the aCC during recent and remote memory consolidation. These observations fit well with a large body of evidence now showing that replay of encoding-related activity during off-line phases of sleep, periods of quiet wakefulness, or conscious recollection are a core mechanism for driving plasticity in hippocampal-cortical networks (Squire and Alvarez, 1995; Frankland and Bontempi, 2005; Walker and Stickgold, 2006; Euston et al., 2007). For instance, repeated activation of hippocampal circuits during slow-wave sleep has been shown to reinstate, in a coordinated and synchronized manner, neuronal activity in a wide set of cortical regions (Ji and Wilson, 2007). Assuming that this phenomenon stabilizes hippocampal-cortical connections and enables a refinement of cortical memory traces, it might explain why information rele- vant to an existing cortical wiring diagram (or mental schema) requires less time to be consolidated. Direct support for this proposal comes from recent data showing that the rate at which systems consolidation occurs in the neocortex is dependent on previously acquired knowledge (Tse et al., 2007).

Collectively, our data show that sequential remodeling within hippocampal-cortical circuits occurs during consolidation of fear memory. Nevertheless, several aspects of this structural reorganization need clarification. For example, the mechanisms controlling the disengagement of the hippocampus remain elusive, although a top-down inhibitory control, presumably arising from cortical regions which are actively engaged in remote memory storage and retrieval, has been hypothesized (Wiltgen et al., 2004; Frankland and Bontempi, 2005). Also, once established in the cortex, do consolidated memories remain in their original form and retain their precision? Recent data suggest that they do 
not, since rats tested for late retrieval of contextual fear show comparable levels of freezing when exposed to the original or a different context (Winocur et al., 2007). This observation implies that an initially hippocampus-dependent and context-specific memory is gradually transformed into a more gist-like and schematic memory, as embedding into cortical networks occurs over time. This notion is fully consistent with the multiple trace theory, which holds that information is initially processed by the hippocampus and then by neocortical regions where multiple traces of the initial experience are formed (Moscovitch and Umiltà, 1990; Moscovitch, 2005). This theory, however, also assumes that a new hippocampally mediated trace is created each time an old memory is retrieved. Although we did not observe that hippocampal spines were increased at the remote memory time point, new traces based on hippocampal synaptic changes other than spine formation cannot be excluded. In fact, contrasting with the traditional view that changes in synapses may be permanent once consolidation of enduring memories has been achieved (Anagnostaras et al., 2000), several lines of evidence now point to a continual updating of post-transcriptional modifications of proteins to ensure long-term memory storage (Routtenberg and Rekart, 2005). These modifications seem to include phosphorylation of proteins already present at the synapse, as highlighted in a recent study, which shows that persistence of long-term associative memory requires ongoing protein kinase $\mathrm{M}$ zeta enzymatic activity in the cortex (Shema et al., 2007). Accordingly, future studies will tackle the question of cortical networks stability over time and their resistance to perturbations (interference) or extinction. One stake of these studies is that unveiling the dynamics of neuronal circuits mediating remote memory persistence might help in dampening abnormally long-lasting fear memories, especially those generating post-traumatic stress disorder.

\section{References}

Anagnostaras SG, Josselyn SA, Frankland PW, Silva AJ (2000) Computerassisted behavioral assessment of Pavlovian fear conditioning in mice. Learn Mem 7:58-72.

Bailey CH, Kandel ER, Si K (2004) The persistence of long-term memory: a molecular approach to self-sustaining changes in learning-induced synaptic growth. Neuron 44:49-57.

Bontempi B, Laurent-Demir C, Destrade C, Jaffard R (1999) Timedependent reorganization of brain circuitry underlying long-term memory storage. Nature 400:671-675.

Dudai Y (2004) The neurobiology of consolidations, or, how stable is the engram? Annu Rev Psychol 55:51-86.

Euston DR, Tatsuno M, McNaughton BL (2007) Fast-forward playback of recent memory sequences in prefrontal cortex during sleep. Science 318:1147-1150.

Frankland PW, Bontempi B (2005) The organization of recent and remote memories. Nat Rev Neurosci 6:119-130.

Frankland PW, Bontempi B, Talton LE, Kaczmarek L, Silva AJ (2004) The involvement of the anterior cingulate cortex in remote contextual fear memory. Science 304:881-883.

Franklin KBJ, Paxinos G (2001) The mouse brain in stereotaxic coordinates, Ed 2. San Diego: Academic.

Frey U, Morris RG (1998) Synaptic tagging: implications for late maintenance of hippocampal long-term potentiation. Trends Neurosci $21: 181-188$

Gibb R, Kolb B (1998) A method for vibratome sectioning of Golgi-Cox stained whole rat brain. J Neurosci Methods 79:1-4.

Glaser EM, Van der Loos H (1981) Analysis of thick brain sections by obverse-reverse computer microscopy: application of a new, high clarity Golgi-Nissl stain. J Neurosci Methods 4:117-125.

Horner CH, Arbuthnott E (1991) Methods of estimation of spine densityare spines evenly distributed throughout the dendritic field? J Anat 177:179-184
Ji D, Wilson MA (2007) Coordinated memory replay in the visual cortex and hippocampus during sleep. Nat Neurosci 10:100-107.

Kim JJ, Fanselow MS (1992) Modality-specific retrograde amnesia of fear. Science 256:675-677.

Knafo S, Ariav G, Barkai E, Libersat F (2004) Olfactory learning-induced increase in spine density along the apical dendrites of CA1 hippocampal neurons. Hippocampus 14:819-825.

Leuner B, Falduto J, Shors TJ (2003) Associative memory formation increases the observation of dendritic spines in the hippocampus. J Neurosci 23:659-665.

Martin KC, Kosik KS (2002) Synaptic tagging - who's it? Nat Rev Neurosci 3:813-820.

Martin SJ, Clark RE (2007) The rodent hippocampus and spatial memory: from synapses to systems. Cell Mol Life Sci 64:401-431.

Maviel T, Durkin TP, Menzaghi F, Bontempi B (2004) Sites of neocortical reorganization critical for remote spatial memory. Science 305:96-99.

McClelland JL, McNaughton BL, O’Reilly RC (1995) Why there are complementary learning systems in the hippocampus and neocortex: insights from the successes and failures of connectionist models of learning and memory. Psychol Rev 102:419-457.

Moscovitch M, Umiltà C (1990) Modularity and neuropsychology: modules and central processes in attention and memory. In: Modular deficits in alzheimer's disease (Schwartz MF, ed), pp 1-59. Cambridge, MA: MIT/Bradford.

Moscovitch M, Rosenbaum RS, Gilboa A, Addis DR, Westmacott R, Grady C, McAndrews MP, Levine B, Black S, Winocur G, Nadel L (2005) Functional neuroanatomy of remote episodic, semantic and spatial memory: a unified account based on multiple trace theory. J Anat 207:35-66.

Restivo L, Roman FS, Ammassari-Teule M, Marchetti E (2006) Simultaneous olfactory discrimination elicits a strain-specific increase in dendritic spines in the hippocampus of inbred mice. Hippocampus 16:472-479.

Rosenbaum RS, Priselac S, Köhler S, Black SE, Gao F, Nadel L, Moscovitch M (2000) Remote spatial memory in an amnesic person with extensive bilateral hippocampal lesions. Nat Neurosci 3:1044-1048.

Ross RS, Eichenbaum H (2006) Dynamics of hippocampal and cortical activation during consolidation of a nonspatial memory. J Neurosci 26:4852-4859.

Routtenberg A, Rekart JL (2005) Post-translational protein modification as the substrate for long-lasting memory. Trends Neurosci 28:12-19.

Routtenberg A, Cantallops I, Zaffuto S, Serrano P, Namgung U (2000) Enhanced learning after genetic overexpression of a brain growth protein. Proc Natl Acad Sci U S A 97:7657-7662.

Shema R, Sacktor TC, Dudai Y (2007) Rapid erasure of long-term memory associations in the cortex by an inhibitor of PKM zeta. Science 317:951-953.

Simons JS, Spiers HJ (2003) Prefrontal and medial temporal lobe interactions in long-term memory. Nat Rev Neurosci 4:637-648.

Squire LR, Alvarez P (1995) Retrograde amnesia and memory consolidation: a neurobiological perspective. Curr Opin Neurobiol 5:169-177.

Squire LR, Bayley PJ (2007) The neuroscience of remote memory. Curr Opin Neurobiol 17:185-196.

Squire LR, Stark CE, Clark RE (2004) The medial temporal lobe. Annu Rev Neurosci 27:279-306.

Takehara-Nishiuchi K, Nakao K, Kawahara S, Matsuki N, Kirino Y (2006) Systems consolidation requires postlearning activation of NMDA receptors in the medial prefrontal cortex in trace eyeblink conditioning. J Neurosci 26:5049-5058.

Teng E, Squire LR (1999) Memory for places learned long ago is intact after hippocampal damage. Nature 400:675-677.

Tse D, Langston RF, Kakeyama M, Bethus I, Spooner PA, Wood ER, Witter MP, Morris RG (2007) Schemas and memory consolidation. Science 316:76-82.

Walker MP, Stickgold R (2006) Sleep, memory, and plasticity. Annu Rev Psychol 57:139-166.

Wiltgen BJ, Brown RA, Talton LE, Silva AJ (2004) New circuits for old memories: the role of the neocortex in consolidation. Neuron 44:101-108.

Winocur G, Moscovitch M, Sekeres M (2007) Memory consolidation or transformation: context manipulation and hippocampal representations of memory. Nat Neurosci 10:555-557.

Zola-Morgan SM, Squire LR (1990) The primate hippocampal formation: evidence for a time-limited role in memory storage. Science 250:288 -290. 